Z. klin. Chem. u. klin. Biochem.

7. Jg., S. 63-65, Januar 1969

\title{
Quantitative Serumproteinveränderungen nach Einfrieren und Auftauen
}

Von G. COHNEN und D. PAar

\author{
Aus dem Zentrallaboratorium der Medizinischen Klinik und Poliklinik (Direktor: Prof. Dr. O. H. Arnold) des Klinikum \\ Essen der Rubr-Universität
}

(Eingegangen am 30. Oktober 1968)

Es wird über Proteinveränderungen in Serumproben nach 20 monatiger Lagerung bei $-28^{\circ}$ und nach wiederholtem Einfrieren und Auftauen berichtet. Die Gesamteiweißkonzentrationen und papierelektrophoretischen Fraktionen zeigten keine wesentliche Änderung. Demgegenüber fielen die meisten, mit radialer Immunodiffusion bestimmten Einzelproteine nach den verschiedenen Gefriermaßnahmen signifikant ab. Als Ursache der Konzentrationsabnahme wird ein Verlust antigener Determinanten durch physikalische und chemische Einflüsse diskutiert. Das Mitführen von Kontrollseren bei langfristiger Lagerung von Serumproben in tiefgefrorenem Zustand erscheint auf Grund der beobachteten Veränderungen empfehlenswert.

\section{Quantitative changes in the serum proteins after freezing and thasing}

Changes in the proteins of serum samples after storage for 20 months at $-28^{\circ} \mathrm{C}$, and after repeated freezing and thawing are reported. The total concentration of protein and of the fractions in paper electrophoresis show essentially no change. On the other hand, most of the individual proteins determined by radial immunodiffusion show a significant decrease after the different freezing treatments. This decrease in concentration is probably caused by a loss of antigenic determinants by physical and chemical effects. On the basis of these observed changes, it is recommended that control sera should be used in conjunction with the investigation of serum samples that are stored deep frozen for long periods.

Neben chemischen und biologischen Einflüssen können physikalische Vorgänge, z. B. Erhitzen und Gefrieren, zu einer Denaturierung von Proteinen führen $(1,2,3)$. Mit dem Verlust der Nativstruktur der Proteine ändern sich auch die immunologischen Eigenschaften und Merkmale (4). So wurden als Folge einer Gefrierdenaturierung bei immunelektrophoretischen Untersuchungen von Serumproben Verminderungen und Verluste von Präzipitationslinien nach verschiedenen Gefriermaßnahmen beschrieben (5).

Ausgehend von diesen Beobachtungen berichtet die vorliegende Mitteilung über quantitative Bestimmungen von Proteinen in Seren, die über einen längeren Zeitraum in tiefgefrorenem Zustand aufbewahrt bzw. wiederholt eingefroren und aufgetaut wurden.

\section{Material und Methodik}

Als Untersucbungsmaterial diente Kubitalvenenblut von zwei Gruppen mit je 10 nüchternen Normalpersonen. Das Serum wurde durch Zentrifugieren (15 Min. bei $1500 \mathrm{~g}$ ) gewonnen. Die Bestimmung der Ausgangskonzentrationen der Proteine erfolgte in den einzelnen Serumproben innerhalb von 3 Stdn. nach Blutentnahme. Anschließend wurden die Proben beider Gruppen in Einzelportionen $\mathrm{zu} 2 \mathrm{ml}$ in Zentrifugenröhrchen (Durchmesser $13 \mathrm{~mm}$, Länge $100 \mathrm{~mm}$ ), mit Parafilm verschlossen, bei $-28^{\circ}$ in einem Gefrierschrank ${ }^{1}$ ) eingefroren. Die weitere Behandlung der Seren war unterschiedlich.

Serumproben Gruppe I: In diesen zehn Seren wurden die Proteine nach 20 monatiger Aufbewahrung bei $-28^{\circ}$ erneut bestimmt.

Serumproben Gruppe II: Jede Probe dieser zehn.Seren wurde in 4 Einzelportionen aufgeteilt. Sämtliche Einzelportionen wurden täglich einmal unter standardisierten Bedingungen (Auftauzeit $1 \mathrm{Std}$. bei $21^{\circ}$ ) aufgetaut und anschließend wieder bei $-28^{\circ}$ eingefroren. Die Serumproteinbestimmungen erfolgten in der ersten Einzelportion am 4., in der zweiten am 8., in der dritten am 12. und in der vierten am 16. Tag nach Versuchsbeginn. Vor der Untersuchung wurden die Serumproben beider Gruppen gründlich mit Hilfe eines Rotamixers (Hook \& Tucker Ltd.) gemischt, um

2) BBC Modell GfS 500, 6063-Din 8953 GN.
Fehlbestimmungen durch unterschiedliche Eiweißsedimentation (6) zu vermeiden.

Die Proteinbestimmungen erfolgten mit folgenden Methoden: Gesamteiweiß (7) auf dem Auto-Analyzer (Technicon), Papierelektrophorese (8) mit automatischem Extinktions- und Integralschreiber (Carl Zeiss) nach Anfärben mit Amidoschwarz $10 \mathrm{~B}$; Einzelproteine mit modifizierter radialer Immunodiffusion (9) unter Verwendung von Antiseren der Behringwerke (Marburg/ Lahn). Bei den untersuchten Einzelproteinen handelte es sich um das saure $\alpha_{1}$-Glykoprotein, $\alpha_{1}$-Antitrypsin, Haptoglobin, $\alpha_{2}$ Makroglobulin, Transferrin, $\beta$-Lipoprotein, $\gamma \mathrm{A}-, \gamma \mathrm{G}-$ und $\gamma \mathrm{M}$ Globulin.

Die Berechnung der absoluten Konzentrationen der papierelektrophoretischen Fraktionen erfolgte entsprechend dem prozentualen Anteil am Gesamteiweiß. Die immunologisch bestimmten Proteinkonzentrationen wurden in $\mathrm{mg} / 100 \mathrm{ml}$ angegeben. Zur Eichung dienten Verdünnungen eines Standardserum bekannter Proteinkonzentration (Standard Humanserum, Behringwerke, Marburg/Lahn, Op. Nr. 166). Die Konzentrationen für $\alpha_{1}$-Antitrypsin und $\beta$-Lipoprotein wurden in Prozent eines gepoolten Normalserum angegeben. Sämtliche Konzentrationsangaben stellen Mittelwerte aus den Einzelbestimmungen der zehn Serumproben beider Gruppen dar. Die statistische Auswertung erfolgte mit Hilfe des $t$-Tests nach Student. Werte von $P<0,05$ wurden als signifikant angesehen.

\section{Ergebnisse}

Tabelle 1 zeigt die Ergebnisse der Bestimmungen für Gesamteiweiß, Albumin, $\alpha_{1}$-Glöbulin, $\alpha_{2}$-Globulin, $\beta$-Globulin und $\gamma$-Globulin in den Serumproben der ersten Gruppe vor und nach 20 monatiger Aufbewahrung bei $-28^{\circ}$. In Tabelle 2 sind die Resultate der entsprechenden Untersuchungen für die Serumproben der Gruppe II nach mehrfachem Einfrieren und Auftauen dargestellt. Tabelle 3 und 4 geben die Ergebnisse der immunologischen Proteinbestimmungen in beiden Gruppen wieder. In Abbildung 1 sind die Konzentrationen der immunologisch bestimmten Einzelproteine beider Gruppen in Prozent des Ausgangswertes gegenübergestellt. 
Tab. 1

Konzentrationen von Gesamteiweiß und papierelektrophoretisclien Folion Aufbewahrung bei $-28^{\circ}$

\begin{tabular}{|c|c|c|c|}
\hline \multicolumn{2}{|c|}{ Untersuchung } & \multirow{2}{*}{$\begin{array}{c}\begin{array}{c}\text { Ausgangswert } \\
\mathrm{g} / 100 \mathrm{ml}\end{array} \\
6,93 \\
0,373\end{array}$} & \multirow{2}{*}{ 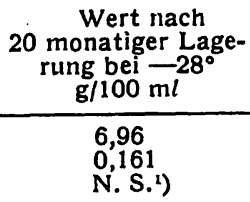 } \\
\hline Gesamteiweiß & $\begin{array}{l}\bar{X} \\
S \\
P\end{array}$ & & \\
\hline Albumin & $\begin{array}{l}\bar{X} \\
S \\
P\end{array}$ & $\begin{array}{l}3,99 \\
0,267\end{array}$ & $\begin{array}{l}4,03 \\
0,231 \\
\text { N. S. }\end{array}$ \\
\hline$a_{1}$-Globulin & $\begin{array}{l}\bar{X} \\
\mathrm{~S} \\
\mathrm{P}<\end{array}$ & $\begin{array}{l}0,360 \\
0,044\end{array}$ & $\begin{array}{l}0,376 \\
0,049 \\
\text { N.S. }\end{array}$ \\
\hline$\alpha_{2}$-Globulin & 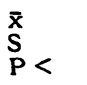 & $\begin{array}{l}0,561 \\
0,076\end{array}$ & $\begin{array}{l}0,543 \\
0,092 \\
\text { N.S. }\end{array}$ \\
\hline$\beta$-Globulin & $\begin{array}{l}\bar{x} \\
S \\
P\end{array}$ & $\begin{array}{l}0,769 \\
0,111\end{array}$ & $\begin{array}{l}0,745 \\
0,104 \\
\text { N. S. }\end{array}$ \\
\hline$\gamma$-Globulin & $\begin{array}{l}\bar{X} \\
\mathbf{S} \\
\mathrm{P}<\end{array}$ & $\begin{array}{l}1,25 \\
0,124\end{array}$ & $\begin{array}{l}1,24 \\
0,173 \\
\text { N. S. }\end{array}$ \\
\hline
\end{tabular}

1) N. S. = nicht signifikant.

Tab. 2

Konzentrationen von Gesamteiweiß und papierelektrophoretischen Fraktionen vor und nach wiederholtem Einfrieren und Auftauen

\begin{tabular}{|c|c|c|c|c|c|c|}
\hline \multicolumn{2}{|c|}{ Untersuchung } & \multirow{2}{*}{$\begin{array}{c}\begin{array}{c}\text { Ausgangswert } \\
\mathrm{g} / 100 \mathrm{ml}\end{array} \\
6,60 \\
0,516\end{array}$} & \multicolumn{4}{|c|}{$\begin{array}{l}\text { Werte nach wiederholtem } \\
\text { Einfrieren und Auftauen } \\
4 \mathrm{mal} 8 \mathrm{~g} / 100 \mathrm{ml} \text {. } 12 \mathrm{mal} 16 \mathrm{mal}\end{array}$} \\
\hline Gesamteiweiß & $\begin{array}{l}\overline{\mathbf{X}} \\
\mathbf{S} \\
\mathrm{P}_{\mathrm{i}}<\end{array}$ & & $\begin{array}{l}6,49 \\
0,581 \\
\text { N.S. }\end{array}$ & $\begin{array}{l}6,65 \\
0,390 \\
\text { N.S. }\end{array}$ & $\begin{array}{l}6,59 \\
0,614 \\
\text { N.S. }\end{array}$ & $\begin{array}{l}6,67 \\
0,683 \\
\text { N. S. }\end{array}$ \\
\hline Albumin & $\begin{array}{l}\bar{x} \\
\mathrm{~S} \\
\mathrm{P}<\end{array}$ & $\begin{array}{l}3,90 \\
0,420\end{array}$ & $\begin{array}{l}3,93 \\
0,338 \\
\text { N. S. }\end{array}$ & $\begin{array}{l}3,95 \\
0,390 \\
\text { N. S. }\end{array}$ & $\begin{array}{l}3,90 \\
0,550 \\
\text { N. S. }\end{array}$ & $\begin{array}{l}3,90 \\
0,442 \\
\text { N. S. }\end{array}$ \\
\hline$\alpha_{2}$-Globulin & $\begin{array}{l}\bar{x} \\
\text { S } \\
P\end{array}$ & $\begin{array}{l}0,310 \\
0,044\end{array}$ & $\begin{array}{l}0,325 . \\
0,053 \\
\text { N. S. }\end{array}$ & $\begin{array}{l}0,299 \\
0,047 \\
\text { N. S. }\end{array}$ & $\begin{array}{l}0,310 \\
0,044 \\
\text { N.S. }\end{array}$ & $\begin{array}{l}0,347 \\
0,088 \\
\text { N.S. }\end{array}$ \\
\hline$\alpha_{2}-$ Globulin & $\begin{array}{l}\bar{X} \\
\text { S } \\
P\end{array}$ & $\begin{array}{l}0,548 \\
0,099\end{array}$ & $\begin{array}{l}0,526 \\
0,094 \\
\text { N.S. }\end{array}$ & $\begin{array}{l}0,585 \\
0,098 \\
\text { N.S. }\end{array}$ & $\begin{array}{l}0,587 \\
0,114 \\
\text { N. S. }\end{array}$ & $\begin{array}{l}0,567 \\
0,131 \\
\text { N.S. }\end{array}$ \\
\hline$\beta$-Globulin & $\begin{array}{l}\overline{\mathbf{x}} \\
\mathrm{S} \\
\mathrm{P}<\end{array}$ & $\begin{array}{l}0,614 \\
0,103\end{array}$ & $\begin{array}{l}0,565 \\
0,106 \\
\text { N. S. }\end{array}$ & $\begin{array}{l}0,658 \\
0,086 \\
\text { N. S. }\end{array}$ & $\begin{array}{l}0,606 \\
0,145 \\
\text { N.S. }\end{array}$ & $\begin{array}{l}0,660 \\
0,168 \\
\text { N.S. }\end{array}$ \\
\hline$\gamma$-Globulin & $\begin{array}{l}\overline{\bar{x}} \\
\mathrm{~S} \\
\mathrm{P}<\end{array}$ & $\begin{array}{l}1,25 \\
0,122\end{array}$ & $\begin{array}{l}1,14 \\
0,107 \\
\text { N.S. }\end{array}$ & $\begin{array}{l}1,164 \\
0,105 \\
\text { N.S. }\end{array}$ & $\begin{array}{l}1,199 \\
0,107 \\
\text { N.S. }\end{array}$ & $\begin{array}{l}1,15 \\
0,172 \\
\text { N. S. }\end{array}$ \\
\hline
\end{tabular}

Tab. 3

Konzentrationen immunologisch bestimmter Serumproteine vor und nach 20 monatiger Aufbewahrung bei $-28^{\circ}$

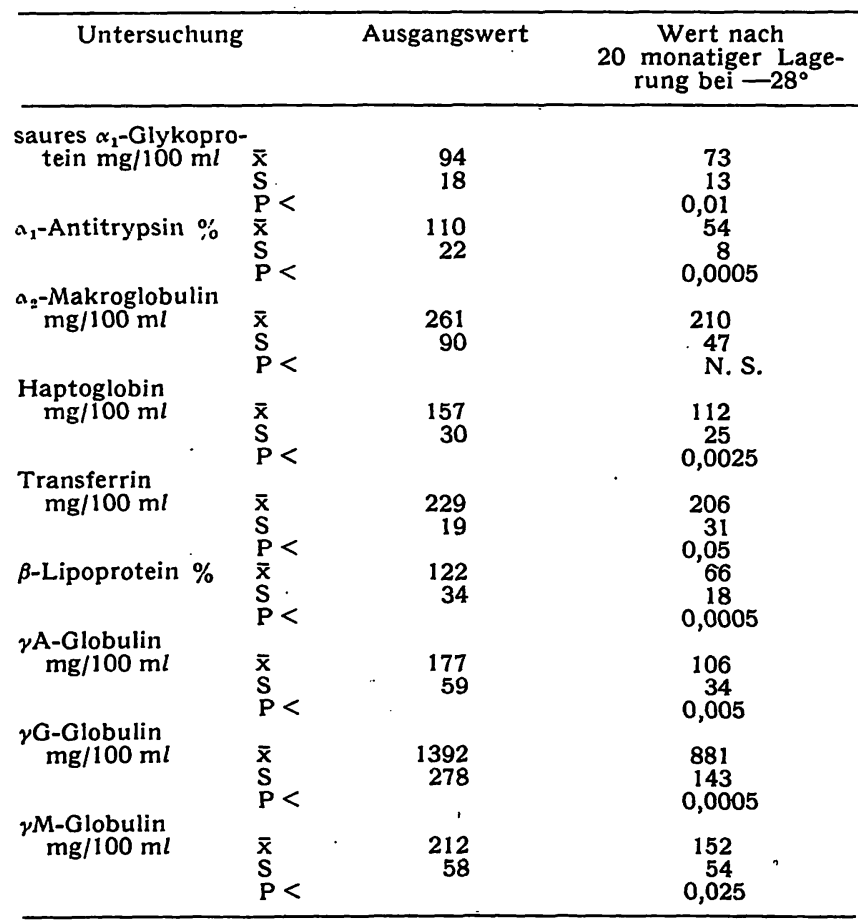

Tab. 4

Konzentrationen immunologisch bestimmter Serumproteine vor und nach wiederholtem Einfrieren und Auftauen

\begin{tabular}{|c|c|c|c|c|c|c|}
\hline \multicolumn{2}{|l|}{ Untersuchung } & $\begin{array}{c}\text { Ausgangs- } \\
\text { wert }\end{array}$ & \multicolumn{4}{|c|}{$\begin{array}{l}\text { Werte nach wiederholtem } \\
\text { Einfrieren und Auftauen } \\
4 \mathrm{mal} \quad 8 \mathrm{mal} 12 \mathrm{mal} 16 \mathrm{mal}\end{array}$} \\
\hline \multicolumn{7}{|c|}{$\begin{array}{l}\text { saures } \alpha_{1} \text {-Glyko- } \\
\text { protein } \mathrm{mg} /\end{array}$} \\
\hline$\alpha_{1}$-Antitrypsin \% & 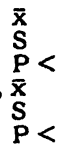 & $\begin{array}{r}127 \\
54 \\
\\
83 \\
28\end{array}$ & $\begin{array}{l}108 \\
45 \\
\text { N. S. } \\
72 . \\
21.5 . \\
\text { N.S. }\end{array}$ & $\begin{array}{l}91 \\
38 \\
\text { N. S. } \\
46 \\
13 \\
0,0025\end{array}$ & $\begin{array}{c}85 \\
32 \\
0,05 \\
43 \\
13 \\
0,0025\end{array}$ & $\begin{array}{c}72 \\
29 \\
0,01 \\
46 \\
1.4 \\
0,0025\end{array}$ \\
\hline $\begin{array}{c}\alpha_{2}-\text { Makroglobulin } \\
\mathrm{mg} / 100 \mathrm{ml}\end{array}$ & $\begin{array}{l}\bar{x} \\
\mathrm{P}\end{array}$ & $\begin{array}{r}283 \\
62\end{array}$ & $\begin{array}{l}273 \\
66 \\
\text { N. S. }\end{array}$ & $\begin{array}{l}250 \\
65 \\
\text { N. S. }\end{array}$ & $\begin{array}{l}221 \\
63 \\
0,05\end{array}$ & $\begin{array}{l}208 \\
54 \\
0,01\end{array}$ \\
\hline $\begin{array}{l}\text { Haptoglobin } \\
\mathrm{mg} / 100 \mathrm{ml}\end{array}$ & 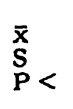 & $\begin{array}{r}227 \\
88\end{array}$ & $\begin{array}{l}150 \\
67 \\
0,05\end{array}$ & $\begin{array}{l}126 \\
39 \\
0,005\end{array}$ & $\begin{array}{c}93 \\
28 \\
0,0005\end{array}$ & $\begin{array}{c}90 \\
31 \\
0,0005\end{array}$ \\
\hline $\begin{array}{l}\text { Transferrin } \\
\text { mg/100 ml } \\
\beta \text {-Lipoprotein \% }\end{array}$ & 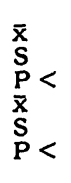 & $\begin{array}{r}276 \\
56 \\
133 \\
32\end{array}$ & $\begin{array}{c}216 \\
50 \\
0,025 \\
118 \\
27 \\
\text { N. S. }\end{array}$ & $\begin{array}{c}193 \\
35 \\
0,0025 \\
98 \\
20 \\
0,01\end{array}$ & $\begin{array}{c}169 \\
31 \\
0,0005 \\
83 \\
18 \\
0,0025\end{array}$ & $\begin{array}{c}150 \\
34 \\
0,0005 \\
67 \\
17 \\
0,0005\end{array}$ \\
\hline $\mathrm{mg} / 100 \mathrm{ml}$ & $\begin{array}{l}\overline{\mathbf{x}} \\
\stackrel{\mathbf{P}}{\mathbf{P}}<\end{array}$ & $\begin{array}{l}248 \\
109\end{array}$ & $\begin{array}{l}237 \\
106 \\
\text { N.S. }\end{array}$ & $\begin{array}{l}226 \\
103 \\
\text { N.S. }\end{array}$ & $\begin{array}{r}155 \\
56 \\
0,025\end{array}$ & $\begin{array}{r}134 \\
49 \\
0,01\end{array}$ \\
\hline $\mathrm{mg} / 100 \mathrm{ml}$ & $\begin{array}{l}\bar{x} \\
\stackrel{P}{P} \\
P\end{array}$ & $\begin{array}{r}1164 \\
190\end{array}$ & $\begin{array}{l}1084 \\
156 \\
\text { N.S. }\end{array}$ & $\begin{array}{l}872 \\
108 \\
0,0025\end{array}$ & $\begin{array}{c}794 \\
116 \\
0,0005\end{array}$ & $\begin{array}{c}751 \\
92 \\
0,0005\end{array}$ \\
\hline $\mathrm{mg} / 100 \mathrm{ml}$ & $\begin{array}{l}\bar{x} \\
\mathbf{S} \\
\mathbf{P}<\end{array}$ & $\begin{array}{r}223 \\
72\end{array}$ & $\begin{array}{l}186 \\
57 \\
\text { N.S. }\end{array}$ & $\begin{array}{l}173 \\
60 \\
\text { N. S. }\end{array}$ & $\begin{array}{l}146 \\
54 \\
0,0125\end{array}$ & $\begin{array}{l}129 \\
45 \\
0,005\end{array}$ \\
\hline
\end{tabular}
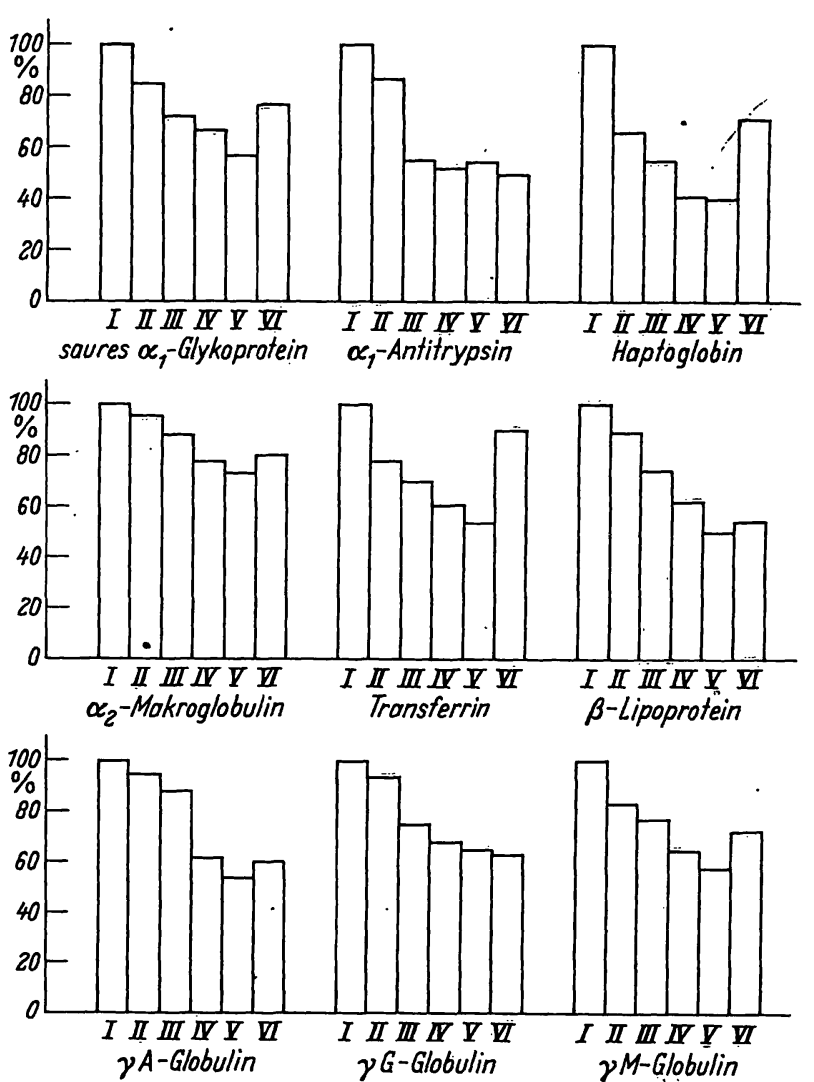

Abb. 1

Gegenüberstellung der immunologisch bestimmten Serumproteinkonzentrationen in Prozent des Ausgangswertes (I) nach 4-(II) 8-(III), 12-(IV) und 16maligem (V) Einfrieren und Auftauen sowie nach 20 Monaten (VI)

\section{Diskussion}

Bei den vorliegenden Untersuchungen konnten weder nach langfristiger Lagerung des Untersuchungsmaterials bei $-28^{\circ}$ (Tab. 1) noch nach wiederholtem Einfrieren und Auftauen der untersuchten Serum- 


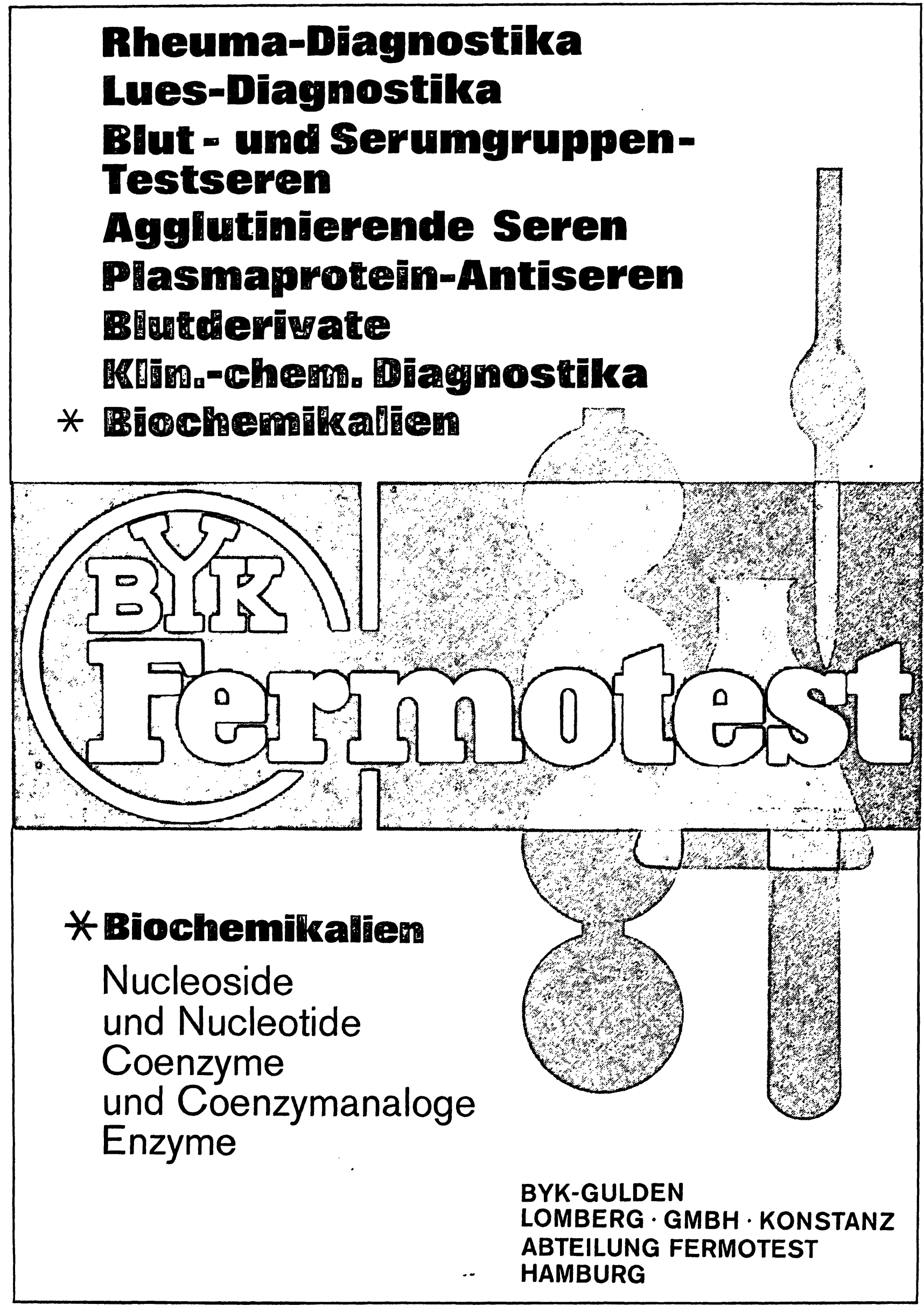




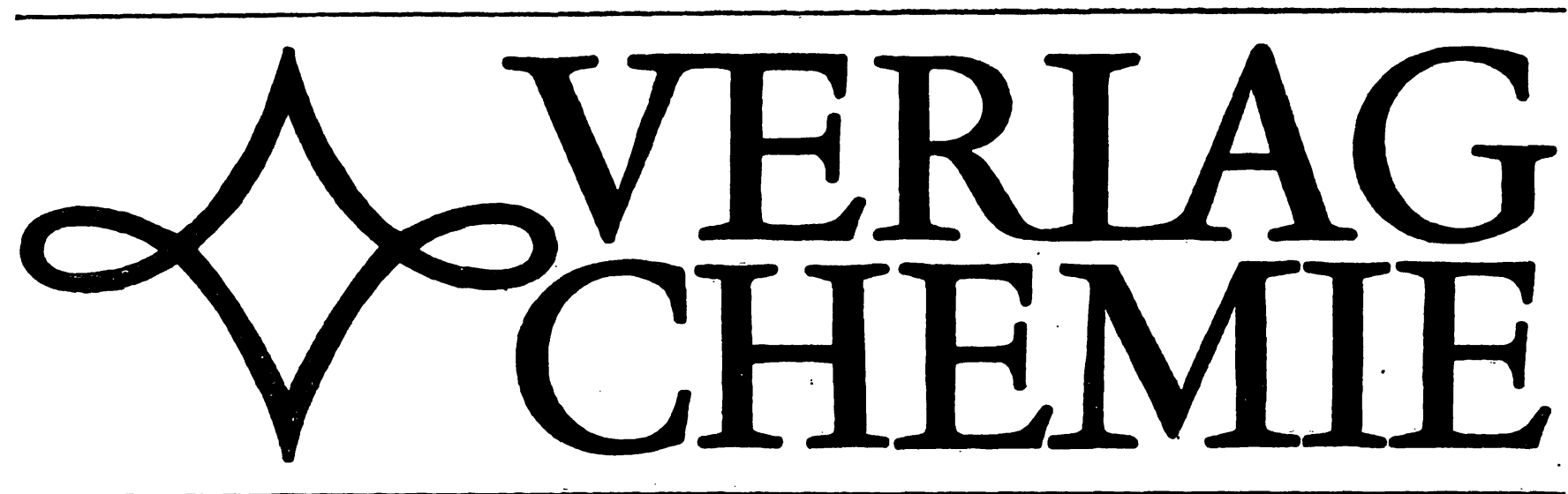

3 Neuauflagen

3 Lebrbücher für den Studenten der Chemie

Walter Ruske

Einführung

1 in die organische

Chemie
Das seit Generationen an den Universitäten eingeführte Lehrbuch von Otto Diels erreichte insgesamt 20 Auflagen. Die 21. von Walter Ruske stark erweiterte und überarbeitete Auflage erschien unter der Autorenbezeichnung „Diels/Ruske“. Aus diesem Standardwerk ist inzwischen ein inhaltlich völlig neues Buch entstanden, für das Walter Ruske allein verantwortlich zeichnet.

1968. XVI, 736 Seiten mit 104 Abb. u. 40 Tab.Ḱ Kunststoffeinband DM 35,-.
F. Albert Cotton

Geoffrey Wilkinson

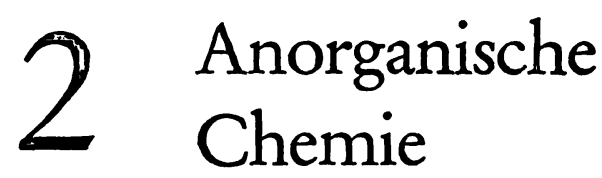

Seit seinem Erscheinen hat der "Cotton/Wilkinson" auch in Deutschland beträchtlichen Anklang, ja begeisterte Aufnahme gefunden. - Das Buch sollte nicht nur in die Hände der Studierenden gelangen, sondern auch von den bereits im Beruf stehenden Chemikern entdeckt werden.

(Kolloid-Zeitschrift)

Nach der 2., überarb. u. erweit. Aufl. von „Advanced Inorganic Chemistry“ übersetzt von Heinz P. Fritz. 1968. 2., verbesserte Aufl. XIV, 1066 Seiten mit 292 Abb. u. 145 Tab. Kunststoffeinband DM 69,-

Man darf wünschen, daß sich das vorliegende Buch in weiterhin zunehmendem $\mathrm{Maß}$ an unseren Hochschulen durchsetzt, zumal es sich gut zum Selbststudium eignet und die Arbeit des oft überlasteten Praktikumsassistenten auf das beste ergänzt.

(Zeitschrift für analytische Chemie)

1968. 3., durchgesebene und ergänzte Aufl. XVI, 422 Seiten mit 82 Abb. u. 16 Mikroaufnabmen. Brosch. DM 28,-

\section{VERLAG CHEMIE - GMBH WEINHEIM/BERGSTR.}


proben (Tab. 2) signifikante Änderungen der Konzentrationen von Gesamteiweiß und papierelektrophoretisch bestimmten Eiweißfraktionen nachgewiesen werden. Diese Ergebnisse stimmen mit den Angaben anderer Autoren für die Lagerungsfähigkeit von tiefgefrorenen Seren $(5,10,11,12)$ und den Einfluß von mehrmaligem Einfrieren und Auftauen auf die Serumproteine $(5,13)$ überein.

Demgegenüber waren bei der quantitativen Bestimmung der Einzelproteine mit radialer Immunodiffusion deutliche Änderungen der ursprünglichen Konzentrationen nachweisbar. Nach 20 monatiger Lagerung des Untersuchungsmaterials bei $-28^{\circ}$ zeigten mit Ausnahme von $\alpha_{2}$-Makroglobulin und Transferrin sämtliche untersuchten Proteine einen deutlich signifikanten Abfall gegenüber den Ausgangswerten (Tab. 3). Diese Ergebnisse unterscheiden sich von den oben erwähnten Beobachtungen (5), bei denen nach 11 monatiger Lagerung der untersuchten Serumproben bei $-20^{\circ}$ immunelektrophoretisch keine auffälligen Änderungen des Serumproteinmusters gefunden wurden. Möglicherweise sind diese Befundabweichungen durch die verschiedenen Lagerungszeiten und -temperaturen sowie durch Einflüsse infolge unterschiedlicher Geschwindigkeit des Einfrier- und Auftauvorganges verursacht. Die Bedeutung dieser Faktoren für Veränderungen in tiefgefrorenem biologischem Material ist bekannt (14, $15,16,17)$. Grundsätzlich kann natürlich auch die unterschiedliche Untersuchungsmethodik eine Rolle spielen. Nach wiederholtem Einfrieren und Auftauen verhielten sich die einzelnen Proteine unterschiedlich (Tab. 4). Die Werte für Haptoglobin und Transferrin fielen bereits nach viermaligem Einfrieren und Auftauen schwach signifikant ab. Nach zwölfmaliger Wiederholung des Einfrier- und Auftauvorganges trat eine signifikante Konzentrationsabnahme sämtlicher bestimmten Proteine auf. Die erwähnten immunelektrophoretischen Untersuchungen (5) ließen ebenfalls nach 12maligem Einfrieren und Auftauen eine Verminderung von Transferrin, $\gamma \mathrm{A}-, \gamma \mathrm{M}$ - und vereinzelt $\gamma$ G-Globulin erkennen. In 2 Fällen fanden sich zusätzlich nicht sicher identifizierbare Präzipitationsdefekte im Bereich der $\alpha$-Globuline, die von den Autoren auf eine mögliche Verminderung von $\alpha_{2}$-Makroglo- bulin zurückgeführt wurden. Bei den vorliegenden Untersuchungen fielen jedoch nicht nur das $\alpha_{2}$-Makroglobulin, sondern auch das im $\alpha$-Globulinbereich wandernde $\alpha_{1}$-Antitrypsin und Haptoglobin signifikant ab.

Die immunologisch nachgewiesenen Proteinverminderungen deuten auf eine Abnahme von antigenen Merkmalen während der verschiedenen Gefriermaßnahmen hin. Als Ursache für den Konzentrationsabfall der Einzelproteine nach 20 monatiger Lagerung bei $-28^{\circ}$ sind, in Übereinstimmung mit den Ergebnissen anderer Autoren (5, 14, 15, 18), physikalische Einflüsse im Sinne einer Gefrierdenaturierung anzunehmen. Hierbei ist ein Verlust von immunologischen Eigenschaften durch Dehydratationsvorgänge mit Anstieg der Ionenkonzentration in den untersuchten Proben zu diskutieren.

Bei der Konzentrationsabnahme der Einzelproteine nach wiederholtem Einfrieren und Auftauen müssen zusätzliche Faktoren berücksichtigt werden. So ist zum Beispiel die für die $\gamma \mathrm{G}$-Globuline unter ähnlichen Bedingungen nachgewiesene Aggregatbildung zu erwähnen $(19,20)$. Auch ein Verlust antigener Determinanten durch proteolytische Vorgänge ist denkbar. Hierbei müßte eine Aktivierung proteolytischer Enzyme durch den häufigen Temperaturwechsel angenommen werden.

Für das Vorliegen unterschiedlicher Einflüsse bei den verschiedenen Gefriermaßnahmen spricht auch das Verhalten von saurem $\alpha_{1}$-Glykoprotein, Haptoglobin und Transferrin (Abb. 1). Diese Proteine zeigten im Gegensatz zu den übrigen untersuchten Serumeiweißen nach 20 monatiger Lagerung im tiefgefrorenen Zustand einen geringeren Konzentrationsabfall als nach 16maligem Einfrieren und Auftauen. Der signifikante Abfall der meisten Einzelproteine nach 20 monatiger Lagerung bei $-28^{\circ}$ wirft die Frage auf, ob nicht grundsätzlich bei langfristiger Aufbewahrung tiefgefrorener Serumproben ein bereits vor dem Einfrieren analysiertes Serum als Kontrollprobe mitgeführt werden sollte. Durch einen Vergleich der Proteinkonzentrationen in diesem Kontrollserum vor und nach dem Einfrieren könnten stärkere Denaturierungseinflüsse während der Lagerung erkannt werden.

\section{Literatur}

1. Fasold, H. und F. Turba, Denaturierung von Proteinen; Wissenschaftl. Veröffentl. d. Dtsch. Gesellschaft für Ernährung, 5, 1, Dr. D. Steinkopf Verlag, Darmstadt (1960). - 2. Wurrmann, F. und H. H. Märkr, Dysproteinämien und Paraproteinämien, 4. Aufl., S. 32, Schwabe-Verlag, Basel-Stuttgart (1963). - 3. Schultze, H. E. und J. F. Hrremans, Molecular Biology of Human Proteins, Vol. I. Nature and Metabolism of Extracellular Proteins, S. 41, Elsevier Publishing Company, AmsterdamLondon-New York (1966). - 4. Arat, F., Nature London 212, 848 (1966). - 5. Scheiffarth, F., H. Götz und R. CERNY: diese Z. 3, 81 (1965). - 6. CASTro, A. und A. Ehrlich, Transfusion 6, 594 (1966). - 7. FAILING, J. F. jr., M. W. BuckLEY und B. ZAK, Amer. J. clin. Path., 33, 83 (1960). - 8. GrassmanN, W., K. HANNIG und M. KNEDEL, Dtsch. med. Wschr. 76, 333 (1951). -
9. WERnER, M., J. Chromatogr. 28,59 (1967). - 10. WALFord, R. L., M. Sowa und D. Daley, Amer. J. clin. Path. 26, 376 (1956). 11. Levey, S. und E. R. Jennings, Amer. J. clin. Path. 20, 1059 (1950). - 12. Strumia, M. M., J. J. McGrai jr. und G. E. Heggestad, Amer. J. clin. Path. 22, 313 (1952): - 13. Mathews, J. und D. A. ButhalA, Amer. J. vet. Res., 17, 485 (1956). - 14. GreIfr, D. und R. T. Kelly, Cryobiol., 2, 335 (1966). - 15. Chrtson, O. P., L. A. Costello und N. O. Kaplan, Federation Proc. 24, Suppl. 15, S. 55 (1965). - 16. Davies, D. F., Federation Proc. 24, Suppl. 15, S. 249 (1965). - 17. MERYMan, H. T., Science, Washington 124, 515 (1956). - 18. Pennell, R. B., Federation Proc., 24, Suppl. 15, S. 269 (1965). - 19. Hansson, Ü.-B., Acta chem. scand. 22, 483 (1968). - 20. Hansson, U.-B., Acta chem. scand. 22, 490 (1968).

Dr. G. Cohnen und Dr. D. Paar 4300 Essen-Holsterhausen Hufelandstr. 55 